\title{
RANDOM LINEAR FUNCTIONALS
}

\author{
BY \\ R. M. DUDLEY $\left({ }^{1}\right)$
}

Let $S$ be a real linear space (=vector space). Let $S^{a}$ be the linear space of all linear functionals on $S$ (not just continuous ones even if $S$ is topological). Let $\mathscr{B}\left(S^{a}, S\right)$ or $\mathscr{B}(S)$ denote the smallest $\sigma$-algebra of subsets of $S^{a}$ such that the evaluation $x \rightarrow x(s)$ is measurable for each $s \in S$. We define a random linear functional (r.l.f.) over $S$ as a probability measure $P$ on $\mathscr{B}(S)$, or more formally as the pair $\left(S^{a}, P\right)$.

In the cases we consider, $S$ is generally infinite-dimensional, and in some ways $S^{a}$ is too large for convenient handling. Various alternate characterizations of r.l.f.'s are useful and will be discussed in $\$ 1$ below.

If $T$ is a topological linear space let $T^{\prime}$ be the topological dual space of all continuous linear functions on $T$. If $T^{\prime}=S$, then a random linear functional over $S$ defines a "weak distribution" on $T$ in the sense of I. E. Segal [23]. See the discussion of "semimeasures" in $\S 1$ below for the relevant construction.

A central question about an r.l.f. $\left(S^{a}, P\right)$, supposing $S$ is a topological linear space, is whether $P$ gives outer measure 1 to $S^{\prime}$. Then we call the r.l.f. canonical, and as is well known $P$ can be restricted to $S^{\prime}$ suitably (cf. 2.3 below).

For simplicity, suppose that for each $s \in S, \int x(s)^{2} d P(x)<\infty$. Let $C(s)(x)=x(s)$. Then $C$ is a linear operator from $S$ into the Hilbert space $L^{2}\left(S^{a}, P\right)$. One seeks conditions on this operator to make the r.l.f. canonical.

If $S$ itself is a Hilbert space, the fundamental theorem of Sazonov [22] and associated results due to Minlos [20] and others assert that it is sufficient for $C$ to be a Hilbert-Schmidt operator, and that this result is the best possible. If $S$ is a Banach space, it is sufficient for $C$ to be nuclear in the sense of Grothendieck [15]; see 8.5 below. The conjecture that it would suffice for $C^{*} C$ to be nuclear, as if $S$ is Hilbert, is not true even in the Gaussian (mean 0) case, where $\int e^{i x(s)} d P(x) \equiv e^{-Q(s)}, Q$ being a nonnegative definite quadratic form on the Banach space $S$; see the end of $\S 6$ below.

In another paper [8], I have, in effect, studied conditions for a Gaussian r.l.f. over a Banach space to be canonical. It appears that the relevant conditions concern the "size" of $C(M)$, where $M$ is the unit ball of $S$. Here the size may be measured by $\varepsilon$-entropy or by volumes of finite-dimensional projections. Conditions like nuclearity of $C$ seem poorly adapted to give precise results here since they measure the size of $C(M)$ only in certain rather arbitrary directions.

Received by the editors October 25, 1967.

(1) Partially supported by the Alfred P. Sloan Foundation and by the National Science Foundation. 
As would be expected, results for the Gaussian case do not hold in general. We shall see that $C(M)$ as a subset of an abstract Hilbert space is not in general sufficient to determine whether the r.l.f. is canonical or not; a unitary transformation may destroy canonicality ( 8.6 below).

Another and most delicate counterexample ( 8.1 below) shows that a nuclear operator from one Banach space $S$ to another $T$, followed by a continuous linear map of $T$ into (nonintegrable) measurable functions, may fail to define a canonical r.l.f. over $S$.

The paper also contains a good many of the known positive results about r.l.f.'s as well as some new ones. The sections are as follows: 0 . Terminology for topological vector spaces. 1. Alternate characterizations. 2. Continuity of r.l.f.'s. 3. Weak topologies. 4. Decomposability and metric linear spaces. 5. Hilbert spaces. 6. Gaussian r.l.f.'s. 7. R.l.f.'s over $l_{p}$ from independent random variables. 8. Failure of extensions of Sazonov's theorem. 9. $L^{p}$ maps of Banach spaces. 10. Means and translates. 11. Stochastic processes and random distributions.

I am much indebted to J. Feldman, L. LeCam, C. M. Deo, L. Schwartz, L. Gross and others for useful discussions.

0 . Terminology for topological vector spaces. We collect here for easier reference some definitions (most of them in standard use) concerning topological vector spaces.

A topological vector space $S$ is called separated if, equivalently, its topology is $T_{1}$ or Hausdorff or its uniformity is separated. A set $B \subset S$ is called bounded if for every neighborhood $U$ of 0 in $S$ there is an $\alpha>0$ such that $\alpha B \subset U$. It is easily seen that the closure of a bounded set is bounded.

Let $S^{\prime}$ be the topological dual space of $S$. The weak topology $w\left(S, S^{\prime}\right)$ on $S$ is the topology of convergence for each fixed element of $S^{\prime}$. Symmetrically the weak-star topology $w\left(S^{\prime}, S\right)$ on $S^{\prime}$ is the topology of pointwise convergence on $S$. The strong topology on $S^{\prime}$ is the topology of uniform convergence on bounded sets of $S$.

A set $B \subset S$ is called a barrel if it is closed, convex, symmetric, and absorbing, i.e. for each $x \in S, \alpha x \in B$ for some $\alpha>0$. If every barrel is a neighborhood of 0 , $S$ is called barreled (tonnelé).

If $1 \leqq p<\infty, l_{p}$ denotes the Banach space with norm $\|\cdot\|_{p}$ of all sequences $\left\{a_{n}\right\}_{n=1}^{\infty}$ of real numbers such that

$$
\left\|\left\{a_{n}\right\}\right\|_{p}^{p} \equiv \sum\left|a_{n}\right|^{p}<\infty
$$

$l_{\infty}$ denotes the Banach space with norm $\|\cdot\|_{\infty}$ of all bounded sequences of real numbers, where

$$
\left\|\left\{a_{n}\right\}\right\|_{\infty} \equiv \sup \left|a_{n}\right| \text {. }
$$

$c_{0}$ denotes the closed subspace of $l_{\infty}$ consisting of sequences converging to 0 . 
A measure space is a pair $(\Omega, Q)$ where $Q$ is a countably additive nonnegative measure on some $\sigma$-ring of subsets of the set $\Omega$. It is a probability space if $Q(\Omega)=1$. $\mathscr{F}(Q)$ denotes the linear space of measurable real-valued functions on $\Omega$ modulo functions vanishing $Q$-almost everywhere. On $\mathscr{F}(Q)$ we put the topology $\mathscr{T}(Q)$ of convergence in measure on all sets of finite measure. If $Q(\Omega)$ is defined and finite, as usually holds in this paper, then $\mathscr{T}(Q)$ is metrizable and complete, e.g. for the metric

$$
d(f, g)=\int|f(x)-g(x)| /(1+|f(x)-g(x)|) d Q(x) .
$$

$\mathscr{T}(Q)$ is usually not locally convex; if e.g. $Q$ is Lebesgue measure on the unit interval $[0,1]$, the only convex sets in $\mathscr{T}(Q)$ are the empty set and the whole space $\mathscr{F}(Q)$.

For $1 \leqq p<\infty, L^{p}(\Omega, Q)$ or $L^{p}(Q)$ denotes the closed subspace of $\mathscr{F}(Q)$ consisting of all $f$ such that

$$
\|f\|_{p}^{p}=\int|f|^{p} d Q<\infty
$$

a Banach space under the norm $\|\cdot\|_{p}$.

1. Alternate characterizations. Let $S$ be a real linear space. For any $x_{1}, \ldots, x_{n}$ in $S$ and any Borel set $B$ in $n$-dimensional Cartesian space $R^{n}$, let

$$
S^{a}\left(x_{1}, \ldots, x_{n} ; B\right)=\left\{y \in S^{a}:\left\langle y\left(x_{1}\right), \ldots, y\left(x_{n}\right)\right\rangle \in B\right\} .
$$

A set of this form will be called a cylinder set in $S^{a}$, based on the finite-dimensional subspace of $S$ spanned by the $x_{i}$. The class of all cylinder sets forms a Boolean algebra, and the set of those based on a fixed finite-dimensional subspace forms a $\sigma$-algebra.

If for $i=1,2, L_{i}$ are linear maps from $S$ into spaces $\mathscr{F}\left(P_{i}\right)$ over probability spaces $\left(\Omega_{i}, P_{i}\right)$, we shall say $L_{1}$ and $L_{2}$ are equivalent, $L_{1} \approx L_{2}$, if for any positive integer $n$, elements $x_{1}, \ldots, x_{n}$ of $S$, and Borel set $B \subset R^{n}$, the probabilities

$$
P_{i}\left(\left\langle L_{i}\left(x_{1}\right), \ldots, L_{i}\left(x_{n}\right)\right\rangle \in B\right)
$$

are equal for $i=1,2$. This is clearly an equivalence relation.

Given an r.l.f. $P$ over $S$, we define its characteristic function (ch.f.) $\chi$ on $S$ by

$$
\chi(s)=\int_{s^{a}} e^{i y(s)} d P(y) .
$$

For each $n$, and $x_{1}, \ldots, x_{n} \in S$, we have a Borel measure $\mu\left(x_{1}, \ldots, x_{n}\right)(\cdot)$ on $R^{n}$ defined by

$$
\mu\left(x_{1}, \ldots, x_{n}\right)(B)=P\left(S^{a}\left(x_{1}, \ldots, x_{n} ; B\right)\right) .
$$

For any finite-dimensional subspace $F$ of $S$, there is a natural map of $S^{a}$ onto $F^{a}$ by restriction; we call it $\pi_{S F}$. Then we obtain a measure

$$
P_{F}=P \circ \pi_{S F}^{-1} \quad \text { on } F^{a} \text {. }
$$


Given a linear subspace $T$ of $S^{a}, \pi_{S F}$ maps $T$ onto $F^{a}$ for every finite-dimensional subspace $F$ of $S$ if and only if $S$ and $T$ are paired (in duality), i.e. $T$ separates points of $S$. (See $\$ 3$ for a further discussion of paired linear spaces.) If so then by cylinder sets in $T$ we shall mean sets $A \cap T$ where $A$ is a cylinder set in $S^{a}$. Then we define a semimeasure $Q$ on $T$ as a finitely additive probability measure on the algebra of cylinder sets in $T$ which is countably additive on the cylinder sets based on each fixed finite-dimensional subspace of $S$. A r.l.f. $P$ defines a semimeasure $Q$ by

$$
P(A)=Q(A \cap T) \text { for each cylinder set } A \subset S^{a} .
$$

We list various known characterizations of random linear functionals ([6], Cartier [4], Bochner [26], Getoor [13]).

(1.4) THEOREM. Let $S$ be a real linear space. Then a complex-valued function $\chi$ on $S$ is the ch.f. of an r.l.f. if and only if it is positive definite, continuous on finitedimensional subspaces, and $\chi(0)=1$.

Given for each $x_{1}, \ldots, x_{n} \in S$ a Borel probability measure $\mu\left(x_{1}, \ldots, x_{n}\right)(\cdot)$ on $R^{n}$, the $\mu(\cdot)(\cdot)$ belong to an r.l.f. $P$ by (1.1) if and only if

$$
S^{a}\left(x_{1}, \ldots, x_{n} ; B\right)=S^{a}\left(y_{1}, \ldots, y_{m} ; C\right)
$$

always implies

$$
\mu\left(x_{1}, \ldots, x_{n}\right)(B)=\mu\left(y_{1}, \ldots, y_{m}\right)(C) .
$$

Given a Borel probability measure $P_{F}$ on $F^{a}$ for each finite-dimensional linear subspace $F$ of $S$, the $P_{F}$ belong to an r.l.f. $P$ by (1.2) if and only if $F \subset G$ implies $P_{F}=P_{G} \circ \pi_{G F}^{-1}$.

If $S$ and $T$ are paired then (1.3) defines a 1-1 correspondence between r.l.f.'s $P$ over $S$ and semimeasures $Q$ on $T$.

Given an r.l.f. $P$ over $S$, the map $s \rightarrow(\cdot)(s)$ is linear from $S$ into $\mathscr{F}(P)$. Conversely, every linear map from $S$ into an $\mathscr{F}\left(P_{1}\right)$, for any probability measure $P_{1}$, is equivalent to one defined by an r.l.f. in the given way.

We can say informally that an r.l.f. corresponds to an equivalence class of linear maps of $S$ into spaces $\mathscr{F}(P)$, but this raises set-theoretic problems since the entire equivalence class may be a proper class, not a set.

A linear map from $S$ into an $\mathscr{F}(P)$ will be called a version of the corresponding (equivalent) r.l.f. There always exist versions for which $P$ is complete, i.e. $B \subset A$ and $P(A)=0$ imply $P(B)=0$.

2. Continuity of r.l.f.'s. If $S$ is a topological linear space, $L$ is linear from $S$ into some $\mathscr{F}(P)$, and $L \approx M$, then clearly $L$ is continuous (resp. sequentially continuous) for $\mathscr{T}(P)$ if and only if $M$ is likewise. If so, the r.l.f. defined by $L$ will be called continuous (resp. sequentially continuous).

Let $X$ be a topological space and let $\mathscr{P}(X)$ be the set of probability measures in $X$ on the Borel $\sigma$-algebra generated by the open sets. Let $T W^{*}$ be the weak-star 
topology on $\mathscr{P}(X)$, i.e. the weakest topology such that $\mu \rightarrow \int f d \mu$ is continuous for each $f$ in $\mathscr{C}(X)$, the space of bounded continuous real-valued functions on $X$. If $X$ is metrizable and separable then $T W^{*}$ on $\mathscr{P}(X)$ is metrizable (Varadarajan [25, Corollary, p. 177, Theorem 2.13, p. 188]). The following is largely known (cf. e.g. [24]):

(2.1) THEOREM. Given an r.l.f. over a topological linear space $S$, with ch.f. $\chi$ and associated measures $\mu(\cdot)(\cdot)$ by (1.1), the following are equivalent:

(I) the r.l.f. is continuous,

(II) $x \rightarrow \mu(x)(\cdot)$ is continuous from $S$ into $\left(\mathscr{P}(R), T W^{*}\right)$,

(III) $\chi$ is continuous.

If "continuous" is replaced by "sequentially continuous" in each of (I), (II) and (III), we obtain weaker conditions which are equivalent among themselves.

Proof. (I) $\Rightarrow$ (II) since convergence in probability of random variables implies weak-star convergence of their distributions. Conversely, if (II) holds and $x_{\alpha} \rightarrow x$ in $S$, then $x_{\alpha}-x \rightarrow 0$, and for any $\varepsilon>0$

$$
\mu\left(x_{\alpha}-x\right)((-\varepsilon, \varepsilon)) \rightarrow 1 .
$$

Thus $L\left(x_{\alpha}\right)-L(x)=L\left(x_{\alpha}-x\right) \rightarrow 0$ in probability, so $L\left(x_{\alpha}\right) \rightarrow L(x)$, and the r.l.f. is continuous.

(II) $\Rightarrow$ (III) is immediate. Conversely suppose $\chi$ is continuous and $x_{\alpha} \rightarrow x$ in $S$. Then for any real $b, b x_{\alpha} \rightarrow b x$ and

$$
\chi\left(b x_{\alpha}\right)=\int_{-\infty}^{\infty} e^{i t} \mu\left(b x_{\alpha}\right)(d t)=\int_{-\infty}^{\infty} e^{i b s} \mu\left(x_{\alpha}\right)(d s) \rightarrow \int_{-\infty}^{\infty} e^{i b s} \mu(x)(d s)=\chi(b x) .
$$

Here for sequences we can apply P. Lévy's continuity theorem [19, p. 191, B], finishing the proof for sequential continuity. For topological continuity another method is needed (we shall show after finishing the proof that the Lévy continuity theorem fails for nets).

Let $d$ metrize $T W^{*}$ on $\mathscr{P}(R)$. For $\mu$ in $\mathscr{P}(R)$ let $f$ be its ch.f.

$$
f(t)=\int e^{i x t} \mu(d x)
$$

$f$ with a subscript will be the ch.f. of $\mu$ with the same subscript. If $\mu_{n} \in \mathscr{P}(R)$ and $\delta$ is the unit mass at 0 , then $\mu_{n} \rightarrow \delta$ weak-star if and only if

$$
\lim _{n \rightarrow \infty|x| \leqq 1}\left|f_{n}(x)-1\right|=0
$$

(Loève [19, p. 202, a and p. 211, b, A]). It follows that for any $\alpha>0$ there is a $\beta>0$ such that for any $\mu$ in $\mathscr{P}(R)$,

$$
\sup _{|x| \leqq 1}|f(x)-1|<\beta \quad \text { implies } d(\mu, \delta)<\alpha .
$$


Let $U$ be a neighborhood of 0 in $S$ such that if $u \in U,|\chi(u)-1|<\beta$. Then there is a neighborhood $V$ of 0 in $S$ such that if $v \in V$ and $|b| \leqq 1$, then $b v \in U$. (This follows simply from continuity of scalar multiplication; local convexity is not required.)

Then

$$
\begin{aligned}
|\chi(b v)-1|<\beta, \quad \chi(b v) & =\int_{-\infty}^{\infty} e^{i y} \mu(b v)(d y) \\
& =\int_{-\infty}^{\infty} e^{i b x} \mu(v)(d x)=f_{v}(b)
\end{aligned}
$$

where $f_{v}$ is the ch.f. of $\mu(v)$. Thus $d(\delta, \mu(v))<\alpha$ and (II) holds.

Clearly continuity implies sequential continuity. (Examples showing that sequential continuity is strictly weaker will be given in Theorem 4.5 below.) The proof is complete.

To see that the Lévy continuity theorem need not hold for nets we use the following known fact.

(2.2) Lemma. Let $a_{1}, \ldots, a_{n}$ be any real numbers and $\varepsilon>0$. Then there exist arbitrarily large integers $m$ with

$$
\left|e^{i m a_{j}}-1\right|<\varepsilon \text { for all } j=1, \ldots, n .
$$

Proof. Let $y(m)_{j}=\exp \left(i m a_{j}\right)$. The sequence of points $y(m)$ in the $n$-dimensional torus $T^{n}$ has a cluster point $y$ since $T^{n}$ is compact. Then we can choose $m_{i}, i=1,2$, such that $\left|y\left(m_{i}\right)_{j}-y_{j}\right|<\varepsilon / 2$ for each $j$. We can let $m_{2}-m_{1}$ be arbitrarily large and $m=m_{2}-m_{1}$, giving the desired conclusion.

Now for each finite set $F=\left\{a_{1}, \ldots, a_{n}\right\}$ of real numbers, let $N=\max \left(\left|a_{1}\right|, \ldots,\left|a_{n}\right|\right)$ and choose $m \geqq N$ such that

$$
\left|\exp \left(i m a_{j}\right)-1\right|<1 / N, \quad j=1, \ldots, n .
$$

Let $\mu_{F}$ be a unit point mass at $m$. The set of all finite subsets of $R$ is directed by inclusion. The net of measures $\mu_{F}$ is such that

$$
\lim _{F} f_{F}(x)=\lim _{F} \int e^{i x t} d \mu_{F}(t)=1
$$

for each real $x$, and 1 is the ch.f. of $\delta$. However, if $f$ is continuous with compact support on $R$ and $f(0)=1$, then

$$
\lim _{F} \int f(x) d \mu_{F}(x)=0 \neq \int f(x) d \delta(x)=1 .
$$

Thus $\mu_{F}$ does not converge weak-star to $\delta$, and the Lévy continuity theorem is false for nets.

Let $\left(S^{a}, P\right)$ be an r.l.f. where $S$ is topological. The following is an immediate consequence of a known result in measure theory (Halmos [16, Theorem 17A, p. 75]): 
(2.3) THEOREM. If $S$ and $S^{\prime}$ are paired, the r.l.f. $P$ is canonical if and only if the semimeasure $Q$ on $S^{\prime}$ defined by (1.3) extends to a countably additive probability measure.

It is clear that any canonical r.l.f. is sequentially continuous, but note that a pointwise convergent net of measurable functions need not converge in probability. It is well known that a continuous r.l.f. need not be canonical (cf. $\$ 4$ below).

Here is an example of a canonical r.l.f. which is not continuous. Let $X$ be the space of all countable ordinals with the order topology. Let $S$ be the linear space of all continuous real-valued functions on $X$. We give $S$ the topology of uniform convergence on compact subsets of $X$. Each element of $S^{\prime}$ is given by a finite, signed, regular Borel measure on $X$, concentrated in some countable, compact subset.

For each $f$ in $S$ there is an $\alpha \in X$ such that $f(\beta)$ has the same value, say $E(f)$, for all $\beta \geqq \alpha$ (see e.g. Dugundji [9, III.8.7, p. 81]). $E$ is a discontinuous linear functional on $S$. Let $P$ be the probability measure on $\mathscr{B}(S)$ in $S^{a}$ defined by a unit point mass at $E$. Then the r.l.f. $P$ is not continuous.

To show that $P$ is canonical, suppose $S^{\prime} \subset A \in \mathscr{B}(S)$. Then $A$ is in the $\sigma$-algebra generated by countably many cylinder sets, based on finite-dimensional subspaces spanned by countably many functions $f_{n}, n=1,2, \ldots$ There is a countable ordinal $\alpha$ such that $\beta \geqq \alpha$ implies $f_{n}(\beta)=f_{n}(\alpha)$ for all $n$. Let $Q$ be a unit point mass at $\alpha$. Then $Q \in A$, and $Q\left(f_{n}\right)=E\left(f_{n}\right)$ for all $n$, so $E \in A$ and $P(A)=1$, as desired.

3. Weak topologies. An important class of topologies on linear spaces is the set of "weak" topologies induced by sets of linear functionals. It may, however, be argued that weak convergence of sequences is reasonable while weak convergence of nets is too weak for most purposes. We shall show that r.l.f.'s which are continuous for weak topologies are nearly concentrated in finite-dimensional subspaces. (Both J. Feldman and L. LeCam obtained independent, unpublished proofs of this.) These r.l.f.'s are thus seen to be a very narrow and rather uninteresting class. Later we shall see that weakly sequentially continuous r.l.f.'s are a much more ample class.

We call linear spaces $S$ and $E$ paired if we are given a bilinear function $(\cdot, \cdot)$ from $S \times E$ to the real numbers, which separates points of $S$ and of $E$, i.e. the maps $s \rightarrow(s, \cdot)$ from $S$ into $E^{a}$ and $e \rightarrow(\cdot, e)$ from $E$ into $S^{a}$ are one-to-one.

For any subset $A$ of $S$ we let

$$
A^{\perp}=\{e \in E:(x, e)=0 \text { for all } x \text { in } A\},
$$

and likewise we define $B^{\perp}, B \subset E$.

Given $S$ and $E$ paired, let $w(S, E)$ be the weakest topology on $S$ for which each $(\cdot, e), e \in E$, is continuous. Then $(S, w(S, E))$ is a separated topological linear space. 
(3.1) THEOREM. Suppose $S$ and $E$ are paired real linear spaces. Let $P$ be an r.l.f. over $S$. Then the r.l.f. is continuous on $S$ for $w(S, E)$ if and only if there are finitedimensional subspaces $E_{n}$ of $E$ such that $P^{*}\left(E_{n}\right) \uparrow 1$.

Proof. Suppose the r.l.f. is continuous for $w(S, E)$. Then, given $\varepsilon>0$, there are $e_{1}, \ldots, e_{m}$ in $E$ such that if $\left|\left(x, e_{j}\right)\right|<1, j=1, \ldots, m$, then

$$
P\left(f \in S^{a}:|f(x)|>\varepsilon\right)<\varepsilon .
$$

Let $F$ be the subspace of $E$ spanned by $e_{1}, \ldots, e_{m}$. Then if $x \in F^{\perp}$, we have for any real number $c$

$$
P(f:|f(c x)|>\varepsilon)<\varepsilon
$$

and hence

$$
P(f: f(x)=0) \geqq 1-\varepsilon .
$$

Proceeding inductively, suppose that for every set $Y$ of $n-1$ elements of $F^{\perp}$, $P\left(Y^{\perp}\right) \geqq 1-\varepsilon$. Let

$$
Z=\left\{x_{1}, \ldots, x_{n}\right\} \subset F^{\perp},
$$

and for each real number $b$ let

$$
H_{b}=\left\{x_{1}+b x_{2}, x_{3}, \ldots, x_{n}\right\}^{\perp} .
$$

Then $P\left(H_{b}\right) \geqq 1-\varepsilon$, and if $b \neq c, H_{b} \cap H_{c}=Z^{\perp}$. The uncountably many sets $H_{b} \sim Z^{\perp}$ are disjoint. Thus for some $b, P\left(H_{b} \sim Z^{\perp}\right)=0$ so $P\left(Z^{\perp}\right) \geqq 1-\varepsilon$. Hence $P\left(X^{\perp}\right) \geqq 1-\varepsilon$ whenever $X \subset F^{\perp}$ and $X$ is finite, hence also if $X$ is countable.

Now we prove that $P^{*}(F) \geqq 1-\varepsilon$. Suppose not, so that for some $A$ in $\mathscr{B}\left(S^{a}, S\right)$, disjoint from $F, P(A)>\varepsilon$. The set $A$ is in the $\sigma$-algebra generated by countably many sets $\left\{f: f\left(x_{j}\right) \in B_{j r}\right\}$, where $x_{j} \in S$ and $B_{j r}$ are Borel sets in the real line. Since this $\sigma$-algebra depends only on the (algebraic) linear span of the $x_{j}$, and $F^{\perp}$ has finite codimension, we may assume that $x_{1}, \ldots, x_{k}$ are linearly independent of each other and of $F^{\perp}$ while $x_{j} \in F^{\perp}$ for $j>k$.

Let $Q$ be the mapping of $S^{a}$ into the set $R^{\omega}$ of all sequences of real numbers defined by $Q(f)=\left\{f\left(x_{j}\right)\right\}$. Then $A=Q^{-1}(B)$ for some set $B=Q(A) \subset R^{\omega}$. Since $F$ is disjoint from $Q^{-1}(B), Q(F)$ is disjoint from $B$ and hence $Q^{-1}(Q(F))$ is disjoint from $A$. Now

$$
Q(F)=\left\{\left\{a_{j}\right\}: a_{j}=0, j>k\right\}
$$

so

$$
P\left(Q^{-1}(Q(F))\right)=P\left(\bigcap_{j>k}\left\{x_{j}\right\}^{\perp}\right) \geqq 1-\varepsilon,
$$

a contradiction, and we have proved "only if". 
Conversely suppose $\varepsilon>0$ and $F$ is a finite-dimensional subspace of $E$ with $P^{*}(F) \geqq 1-\varepsilon / 2$. We regard $E$ as a subset of $S^{a}$, with $(s, f)=f(s), f \in S^{a}$. Let $e_{1}, \ldots, e_{n}$ be a basis of $F$ and for $x \in S$ let

$$
\|x\|_{F}=\sup _{j}\left|\left(x, e_{j}\right)\right| \text {. }
$$

Now for some $\delta>0$,

$$
P(\{f:|(x, f)|>\varepsilon\})<\varepsilon \quad \text { if }\|x\|_{F}<\delta .
$$

If not, let (3.2) fail for $x=x_{k},\left\|x_{k}\right\|_{F}<1 / k, k=1,2, \ldots$ Then

$$
\left\{f:\left|\left(x_{k}, f\right)\right|<\varepsilon \text { for } k \text { large enough }\right\}
$$

is measurable and includes $F$. Hence its probability is at least $1-\varepsilon / 2$, so for some $m$,

$$
P\left\{f:\left|\left(x_{k}, f\right)\right|<\varepsilon \text { for all } k \geqq m\right\}>1-\varepsilon,
$$

contradicting falsity of (3.2) and thus proving $w(S, E)$ continuity of the r.l.f. P. Q.E.D.

(3.3) Corollary. Every r.l.f. continuous for a weak topology $w(S, E)$ on $S$ is canonical for $w(S, E)$.

If $S$ is e.g. an infinite-dimensional Banach space and $E$ is its dual space, then $S$ and $E$ are paired and the topological dual space of $(S, w(S, E))$ is $E$ so "canonical" means the same for $w(S, E)$ as for the norm topology. It is easy to construct canonical r.l.f.'s over $S$ which are not $w(S, E)$ continuous. This reverses the situation for metrizable topologies on $S$, where a canonical r.l.f. is always continuous but not vice versa (see the next section).

If $S$ is a topological linear space, then $S$ and $S^{\prime}$ need not be paired if $S$ is not locally convex or not separated. However, we have in general

(3.4) Corollary. An r.l.f. over $S$ continuous for $w\left(S, S^{\prime}\right)$ is canonical.

Proof. Let $T=\left(S^{\prime}\right)^{\perp} \subset S$. Then the quotient linear space $S / T$ and $S^{\prime}$ are paired. If $\chi$ is the ch.f. of the r.l.f. on $S, w\left(S, S^{\prime}\right)$ continuity implies that $\chi$ is constant on each coset of $T$ and hence naturally defines an r.l.f. over $S / T$, continuous for $w\left(S / T, S^{\prime}\right)$. This r.l.f. is canonical by Corollary 3.3 , and hence so is the original one over $S$. Q.E.D.

A weakly sequentially continuous r.l.f. over $S$ also defines naturally an r.l.f. on $S /\left(S^{\prime}\right)^{\perp}$. The following implications between continuity properties of r.l.f.'s over a general topological linear space $S$ have now been proved or are obvious, where "w.s.c." stands for "weakly sequentially continuous," etc.:

$$
\text { w.c. }\left\{\begin{array}{c}
\Rightarrow \text { canonical } \Rightarrow \text { w.s.c. } \Rightarrow \\
\Rightarrow \text { continuous } \Rightarrow
\end{array}\right\} \text { s.c. }
$$


Examples of Gaussian r.l.f.'s over Hilbert space, to be given in $\S 5$ below, and the example given at the end of $\S 2$ above show that only the implications given in (3.5) hold in general.

4. Decomposability and metric linear spaces. Let $S$ be a topological linear space and $L$ a linear map of $S$ into $\mathscr{F}(Q)$ for some measure space $(\Omega, Q)$. We call $L$ decomposable if there is a map $M$ from $S$ into measurable functions on $\Omega$ such that the following two conditions hold:

(a) for each $s$ in $S, M(s)=L(s)$ almost surely,

(b) for almost all $\omega, s \rightarrow M(s)(\omega)$ is a continuous linear functional on $S$.

It is easily seen that an r.l.f. is canonical if and only if it has at least one decomposable version, e.g. with $\Omega=S^{\prime}$.

The r.l.f. defined at the end of $\S 2$ is canonical. It has a version $L$ defined for a probability space $\Omega$ containing only one point, and such a version is not decomposable. Thus the hypotheses of metrizability and separability cannot simply both be dropped from the following result (in [24], Theorem 2 is false).

(4.1) THEOREM. Suppose given a separable metric linear space $S$ and a canonical r.l.f. over S. Then every version $L$ of this r.l.f. is decomposable.

Proof. Since $S$ is metrizable, its topology is metrized by a metric $d$ such that $d(x, y)=d(x-y, 0)$ for all $x$ and $y$ in $S[17$, Theorem 8.3, p. 70]. Also, $S$ has a countable subset $T=\left\{x_{n}\right\}_{n=1}^{\infty}$ whose elements are linearly independent and whose linear span is dense in $S$.

For any positive integer $n$, rational numbers $a_{1}, \ldots, a_{n}$, and $\omega \in \Omega$, let

$$
L_{\omega}\left(\sum_{j=1}^{n} a_{j} x_{j}\right)=\sum a_{j} L\left(x_{j}\right)(\omega) .
$$

(We take a definite value of $L\left(x_{j}\right)(\omega)$ for all $\omega$. In general an equation such as $L(x+y)=L(x)+L(y)$ holds only almost everywhere, the exceptional set depending on $x$ and $y$.)

Let $A$ be the countable domain of each $L_{\omega}$ as just defined. Then $A$ is dense in $S$, $L_{\omega}$ is linear on $A$ over the rational numbers, and for each $y$ in $A, L_{\omega}(y)=L(y)(\omega)$ for almost all $\omega$.

For any positive integers $j$ and $r$ let $A_{j r}$ be the set of all $\omega$ such that for some $x$ in $A, d(0, x)<1 / j$ and $\left|L_{\omega}(x)\right|>1 / r$. Then for each fixed $r$

$$
P\left(\bigcap_{j=1}^{\infty} A_{j r}\right)=0
$$

since $A$ is countable and $L$ is canonical. Thus with probability $1, L_{\omega}$ is uniformly continuous on $A$ and hence has a (unique) extension to an element $L_{\omega}$ of $S^{\prime}$. Since $L$ is canonical, it is sequentially continuous, hence continuous since $S$ is metrizable. Thus for each $x$ in $S, L(x)(\omega)=L_{\omega}(x)$ for almost all $\omega$. Letting $M(x)(\omega)$ $=L_{\omega}(x)$ we see that $L$ is decomposable. Q.E.D. 
The hypotheses on $S$ in the above theorem are equivalent to the assumption that its topology has a countable base. This can be weakened; for example, $S$ can be taken as a strict inductive limit of separable metric linear spaces.

5. Hilbert spaces. We begin with a discussion of Gaussian r.l.f.'s which shows that none of the upper three implications in (3.5) is reversible. Most of the facts collected here are known.

A bounded linear operator $A$ from a Hilbert space $H$ to another is called a Hilbert-Schmidt operator if $\sum\left\|A f_{n}\right\|^{2}<\infty$ for some (and then every) orthonormal basis $\left\{f_{n}\right\}$ of $H[12$, I.2.2].

(5.1) THEOREM. Let $H$ be a Hilbert space. For any linear operator $A$ from $H$ into itself, the function $\chi(x)=\exp \left(-\|A x\|^{2}\right)$ is the ch.f. of an r.l.f. which is

(a) continuous if and only if $A$ is bounded,

(b) weakly sequentially continuous if and only if $A$ is compact,

(c) canonical if and only if $A$ is a Hilbert-Schmidt operator,

(d) weakly continuous if and only if $A$ has finite-dimensional range and is bounded.

Proof. It is well known that $\exp \left(-\|x\|^{2}\right)$ is positive definite, and it follows, e.g. from Theorem 1.4 above, that $\chi$ is the ch.f. of an r.l.f. Let $L$ be a version of it.

For any topology $\mathscr{U}$ making $(H, \mathscr{U})$ a topological vector space, let $C$ be either sequential convergence or convergence of nets for $\mathscr{U}$. Then the following are equivalent:

(I) $L$ is continuous from $(H, C)$ to $(\mathscr{F}(P), \mathscr{T}(P))$,

(II) $\chi$ is continuous from $(H, C)$ to the complex numbers,

(III) $x \rightarrow\|A x\|$ is continuous from $(H, C)$ to the real numbers,

(IV) $A$ is continuous (at 0$)$ from $(H, C)$ to $(H,\|\cdot\|)$.

Now (a) is obvious. (b) is true since $A$ is compact from $H$ into itself if and only if it transforms weakly convergent sequences into strongly convergent ones [21, pp. 206-207]. For (c), see Minlos [20, pp. 311-312]. For (d), it is known and not hard to prove that $A$ is continuous from $H$ with weak topology to $H$ with norm topology if and only if it is bounded and has finite-dimensional range.

5.1(d) was noted previously by L. LeCam. To some extent, 5.1(c) carries over to general (non-Gaussian) r.l.f.'s on a Hilbert space. We state the known facts:

(5.2) THEOREM. Let $H$ be a separable infinite-dimensional Hilbert space, and let $\chi$ be the ch.f. of a r.l.f. over $H$. Then the following are equivalent:

(a) the r.l.f. is canonical,

(b) $\chi$ is continuous (at 0$)$ on $H$ for the weakest topology $\mathscr{T}$ making all HilbertSchmidt operators continuous from $(H, \mathscr{T})$ to $H$ with its norm topology,

(c) there exists a Hilbert-Schmidt operator $A$ and a ch.f. $\mathscr{N}$ of a continuous r.l.f. such that $\chi=\mathscr{N} \circ A$. 
The above theorem was proved by V. Sazonov [22]. The main theorem of Minlos [20] is closely related. A later, shorter proof was given by Kolmogorov [18].

When the r.l.f. $P$ with ch.f. $\mathscr{N}$ is such that for some $K<\infty$

$$
\int f(x)^{2} d P(f) \leqq K\|x\|^{2}
$$

for all $x$ in $H$, the implication (c) $\Rightarrow$ (a) is especially easy to prove. Note that in (c), $A$ may take $H$ into a different Hilbert space.

6. Gaussian r.l.f.'s. A Gaussian r.l.f. (with mean 0) over a linear space $S$ is one with ch.f. of the form $e^{-Q}$ where $Q$ is a nonnegative definite quadratic form on $S$. (Every such $e^{-Q}$ is indeed the ch.f. of an r.l.f. $\left(S^{a}, P\right)$ with any version $L$ satisfying $E L(s)=0$ for all $s \in S$.) Throughout this section, let $L$ be a version of such an r.l.f. over a topological linear space $S$.

Let $B(x, y)=E(L(x) L(y))$. Then $B$ is a symmetric, nonnegative definite bilinear form, or semi-inner product, on $S$. There is a unique linear map $A$ of $S$ into $S^{a}$ such that

$$
A(x)(y)=B(x, y) \text { for all } x, y \in S .
$$

Let $C$ map $S$ (with its given topology) via the identity into the Hilbert space $H$ which is the completion of $S$ for $B$ modulo $\{x: B(x, x)=0\}$. Then the ch.f. of the r.l.f. is given by

$$
E e^{i L(x)}=e^{-B(x, x) / 2}=e^{-A(x)(x) / 2}=e^{-\|C x\|^{2} / 2} .
$$

$C$ has an adjoint $C^{*}$ mapping $H$ (identified with its own dual space) into $S^{a}$, and we have $A=C^{*} C$.

In view of the implications in 3.5 above we can restrict ourselves to sequentially continuous r.l.f.'s in studying the other, stronger continuity conditions. We state first, with the above notations, a fact previously known to L. LeCam.

(6.2) The following are equivalent:

(a) The (Gaussian) r.l.f. is weakly continuous,

(b) $C$ is continuous and has finite-dimensional range,

(c) $A$ and $B$ identify with an element of the algebraic tensor product $S^{\prime} \otimes S^{\prime}$.

Proof. (a) $\Rightarrow$ (b) is proved as 5.1(d). (c) means that we can write

$$
A(x)(y)=B(x, y)=\sum_{i=1}^{n} f_{i}(x) g_{i}(y)
$$

for some finite $n$ and $f_{i}, g_{i} \in S^{\prime}$. Since $B$ is a semi-inner product we can take $f_{i}=g_{i}$. This follows easily from (b). By 6.1 clearly (c) implies (a). Q.E.D.

(6.3) (a) The following are equivalent:

(I) The r.l.f. is continuous.

(II) $C$ is continuous.

(III) $B$ is jointly continuous. 
(b) $A$ is continuous from $S$ to the strong topology of $S^{\prime}$ if and only if $B$ is hypocontinuous (for the family of all bounded sets in $S$ ) [3, Chapitre III, §4, no. 2]. These conditions hold if $B$ is continuous.

(c) B hypocontinuous implies B continuous for $S$ metrizable or barreled or bornologic, but not for all $S$.

Proof. (a) is routine in view of 6.1 and the proof of 5.1. (On a Hilbert space, the inner product is jointly continuous.)

(b) follows directly from Bourbaki's discussion as cited.

For (c), suppose $B$ is hypocontinuous and let $F=\{x: B(x, x) \leqq 1\}$, a convex, circled set.

Then $F$ is closed since if $B(x, x)=c^{2}, c>1$, then for all $y$ in some neighborhood of $x, B(x, y)>c$ and hence $B(y, y)>1$ by the Schwartz inequality. Also $F$ absorbs all bounded sets. Thus $B$ is sequentially continuous, and continuous if $S$ is metrizable, barreled or bornologic.

It is well known that a hypocontinuous bilinear functional need not be continuous. Not having found in the literature an example which is an inner product, I present one here. Let $J$ be an uncountable set and let $S$ be the space of all realvalued functions $f$ on $J$ such that $N(f) \equiv\{x: f(x) \neq 0\}$ is finite. On $S$ we put the topology $\mathscr{T}$ defined by all pseudo-norms

$$
\|f\|_{H}=\sum_{J}|H(x) f(x)|
$$

where $H$ is any real-valued function on $J$ such that $N(H)$ is countable. For each $\mathscr{T}$-bounded set $M \subset S$ there is a finite set $F \subset J$ such that $N(f) \subset F$ for all $f \in M$. Thus if

$$
B(f, g)=\sum_{J} f(x) g(x)
$$

then $B$ is hypocontinuous on $S$, but it is not continuous. Q.E.D.

(6.4) (a) If $C$ is compact, or if $S$ is separated and locally convex and $A$ is compact into strong $S^{\prime}$, then the r.l.f. is weakly sequentially continuous (w.s.c.).

(b) If the r.l.f. is w.s.c. and $S$ is a reflexive Banach space, then $C$ is compact and $A$ is compact into strong $S^{\prime}$.

(c) If e.g. $S=l_{1}$, then $A$ and $C$ need not be compact if the r.l.f. is w.s.c.

Proof. $H$ and strong $S^{\prime}$ are separated locally convex spaces. If $C$ or $A$ is compact, then it is continuous, so if $x_{n} \rightarrow 0$ weakly in $S$, then $C\left(x_{n}\right) \rightarrow 0$ weakly, as does $A\left(x_{n}\right)$. The $C\left(x_{n}\right)$ or $A\left(x_{n}\right)$ all belong to a given strongly compact set, where the weak topology, being separated, equals the stiong topology. Thus $C\left(x_{n}\right)$ or $A\left(x_{n}\right) \rightarrow 0$ strongly. Since $\left\{x_{n}\right\}$ is a bounded set [3, p. 70, Corollaire], $A\left(x_{n}\right)\left(x_{n}\right) \rightarrow 0$, so in either case the r.l.f. is w.s.c.

Conversely suppose the r.l.f. is w.s.c. Then if $x_{n} \rightarrow x$ weakly in $S, C\left(x_{n}\right) \rightarrow C(x)$ strongly in $H$. If $S$ is a reflexive Banach space, then its unit ball $M$ is weakly compact, hence weakly sequentially compact by the Eberlein-Smulian theorem [10, 
V.6.1, p. 430]. Thus $C(M)$ is strongly sequentially compact, i.e. compact, in $H$, so $C$ is a compact operator. Since $C^{*}$ is continuous from $H$ into strong $S^{\prime}, A(M)$ $=C^{*} C(M)$ is compact in strong $S^{\prime}$, and $A$ is compact.

If $S=l_{1}$, let $Y_{n}$ be independent Gaussian random variables with $E Y_{n}=0$ and $E Y_{n}^{2}=1$. Let $L\left(\left\{x_{n}\right\}\right)=\sum x_{n} Y_{n}$. Then $L$ is a version of a continuous r.l.f. on $l_{1}$. A sequence of elements of $l_{1}$ converging weakly to 0 actually converges strongly to 0 [10, IV.8.14, p. 296]. Thus the r.l.f. is w.s.c., but clearly $A$ and $C$ are not compact. Q.E.D.

It remains to discuss conditions on $A, B$ and $C$ for the r.l.f. to be canonical. Here we confine ourselves to showing that a natural generalization of 5.1(c) fails for Banach spaces. Given Banach spaces $S$ and $T$, an operator $A$ from $S$ into $T$ is called nuclear if

$$
A(x)=\sum_{n=1}^{\infty} a_{n} f_{n}(x) t_{n}
$$

where $f_{n} \in S^{\prime},\left\|f_{n}\right\|^{\prime} \leqq 1$, where $\|\cdot\|^{\prime}$ is the usual norm in $S^{\prime},\left\|t_{n}\right\| \leqq 1$, and $\sum\left|a_{n}\right|<\infty$.

Now in the notation we have been using, if $S$ is a Hilbert space the operator $A$ from $S$ into $S^{\prime}$ is nuclear if and only if $C$ is Hilbert-Schmidt (cf. [12, I.2.3., Theorem 4, equation (11)]). Thus in this case the r.l.f. is canonical if and only if $A$ is nuclear.

We shall now show that the latter statement, in particular "if," fails to be true for Banach spaces. (I do not know whether "only if" holds here.) Let $S=c_{0}$. Let $B\left(\left\{x_{n}\right\},\left\{y_{n}\right\}\right)=\sum x_{n} y_{n} / n^{2}$. Then $B$ is an inner product on $c_{0}$ and defines an operator $A$ from $S$ into $S^{\prime}=l_{1}$ which is nuclear. However, the associated Gaussian r.l.f. is not canonical since if $y_{n}$ are independent Gaussian random variables with $E y_{n}=0$ and $E y_{n}^{2}=1 / 2 n^{2}$ then $\sum\left|y_{n}\right|=\infty$ with probability 1 by the three-series theorem [19, p. 237]; cf. [8, Proposition 6.6].

7. R.l.f.'s on $l_{p}$ from independent random variables. Throughout this section let $X_{n}, n=1,2, \ldots$, be independent equidistributed random variables over a probability space $(\Omega, P)$ and $1 \leqq p<\infty$. It is easy to see that $E\left|X_{1}\right|<\infty$ is sufficient for $\left\{a_{n}\right\} \rightarrow \sum a_{n} X_{n}$ to define a continuous r.l.f. over $l_{1}$. We shall explore such situations further.

(7.1) Lemma. Suppose for all $\left\{a_{n}\right\}$ in $l_{p}$, the series $\sum a_{n} X_{n}$ converges in probability. Then the sum $L\left\{a_{n}\right\}$ of the series defines a continuous r.l.f. over $l_{p}$.

Proof. $L$ is a linear map of $l_{p}$ into $\mathscr{F}(P)$. The partial sums of the series are continuous, so $L$ is a Borel measurable homomorphism of a complete metric group into a metric group. Hence it is continuous ( $\mathrm{S}$. Banach [2, Théorème 4, p. 23]). Q.E.D.

We note that a series of independent real-valued random variables is convergent in probability if and only if it converges with probability $1[19, \mathrm{p} .249$, a(i)]. 
Let $F$ be the common distribution function of the $X_{n}$. We call $F p$-compatible if the hypothesis of Lemma 7.1 holds. We call $F$ weakly p-finite if $M^{p} P\left(\left|X_{1}\right| \geqq M\right)$ is uniformly bounded for $0 \leqq M<\infty$. Note that

$$
E\left|X_{1}\right|^{p}<\infty \Rightarrow F \text { weakly } p \text {-finite } \Rightarrow E\left|X_{1}\right|^{p-\delta}<\infty
$$

for all $\delta>0$.

(7.2) THEOREM. The condition

(I) $F$ is p-compatible

is equivalent to the following for the given values of $p$ :

For $2<p<\infty$, (II) $X_{1} \equiv 0$.

For $p=2$, (III) $E X_{1}^{2}<\infty$ and $E X_{1}=0$.

For $1<p<2$, (IV) $F$ is weakly $p$-finite and $E X_{1}=0$.

For $p=1$, (V) $F$ is weakly 1-finite and $\int_{-M}^{M} t d F(t)$ is uniformly bounded for $0 \leqq M<\infty$.

Proof. We repeatedly apply Kolmogorov's three-series theorem [19, p. 237]. For any random variable $Y$ let $Y^{1}=Y$ for $|Y| \leqq 1$ and $Y^{1}=0$ otherwise. Then almost sure convergence of a series $\sum Y_{n}$ of independent random variables is equivalent to simultaneous convergence of the three series

Series A: $\sum P\left(\left|Y_{n}\right| \geqq 1\right)$.

Series B: $\sum E Y_{n}^{1}$.

Series C: $\sum E\left(\left(Y_{n}^{1}\right)^{2}\right)$.

We shall always let $Y_{n}=a_{n} X_{n}$ for suitable real numbers $a_{n}$.

For $2<p<\infty$, clearly (II) $\Rightarrow$ (I). Conversely let $a_{n}=n^{-1 / r}, 2<r<p$, and apply Series $C$ to obtain that either $\sum a_{n}^{2}<\infty$, impossible, or $X_{1} \equiv 0$, i.e. (I) $\Rightarrow$ (II).

Next we gather

(7.3) Lemma. $F$ is not p-compatible under either of the following conditions:

(a) $1 \leqq p \leqq 2$, and $F$ is not weakly $p$-finite.

(b) $p=1$ or 2 , and $\int_{|t| \leqq M} t^{p} d F(t)$ is unbounded as $M \rightarrow \infty$.

Proof. For $n=1,2, \ldots$, choose $M_{n} \geqq n^{2}$ so that, in case (a), $M_{n}^{p} P\left(\left|X_{1}\right| \geqq M_{n}\right) \geqq n$; in case (b), $\int_{|t| \leqq M_{n}} t^{p} d F \geqq n$. (If necessary we replace $X_{j}$ by $-X_{j}$ for all $j$.) Let $a_{j}=1 / M_{n}$ for $k_{n} \leqq j<k_{n+1}$ where $k_{1}=1$ and $k_{n+1}-k_{n} \equiv r_{n}$, an integer such that

$$
1 \leqq n^{2} r_{n} / M_{n}^{p} \leqq 2 \text {. }
$$

Then $\left\{a_{j}\right\} \in l_{p}$. For (a), Series A, $\sum P\left(\left|X_{1}\right| \geqq 1 / a_{j}\right)$ diverges; for (b),

$$
\sum a_{j}^{p} \int_{|t| \leqq 1 / a_{j}} t^{p} d F=\infty
$$

(Series B or C), proving the Lemma.

Thus for $1 \leqq p \leqq 2$, (I) implies that $F$ is weakly $p$-finite and hence, for $p>1, E X_{1}$ exists. Let $a_{n}=1 / n$, so $\left\{a_{n}\right\} \in l_{p}$. Then by Series B, $\sum b_{n} / n$ converges where $b_{n} \rightarrow E X_{1}$, so $E X_{1}=0$. 
Now for $p=2$, (III) $\Rightarrow$ (I) is straightforward. Conversely, assume (I). Then $E X_{1}=0$ and by Lemma 7.3(b) for $p=2, E X_{1}^{2}<\infty$, so (I) $\Rightarrow$ (III).

For $1<p<2$ we have proved (I) $\Rightarrow$ (IV). Conversely, assume (IV), and let $\left\{a_{n}\right\} \in l_{p}$.

Series A: $\sum P\left(\left|X_{1}\right| \geqq 1 /\left|a_{n}\right|\right) \leqq M \sum\left|a_{n}\right|^{p}<\infty$ for some $M>0$.

Series B: $\sum E\left(\left(a_{n} X_{n}\right)^{1}\right)=-\sum a_{n} \int_{|t|>1 /\left|a_{n}\right|} t d F(t)$.

It suffices to consider the series

$$
\begin{aligned}
\sum \mid a_{n} & \int_{1 /\left|a_{n}\right|}^{\infty} t d(F(t)-1) \mid \\
& \left.=\sum_{n} \mid a_{n}\{t(F(t)-1)]_{1 /\left|a_{n}\right|}^{\infty}-\int_{1 /\left|a_{n}\right|}^{\infty}(F(t)-1) d t\right\} \mid \\
& \leqq M \sum_{n}\left|a_{n}\right|\left(\left|a_{n}\right|^{p-1}+\int_{1 /\left|a_{n}\right|}^{\infty} d t /|t|^{p}\right) \leqq K \sum_{n}\left|a_{n}\right|^{p}<\infty
\end{aligned}
$$

for some $K>0$.

Series C: $\sum_{n} E\left(\left(\left(a_{n} X_{n}\right)^{1}\right)^{2}\right)=\sum_{n} a_{n}^{2} \int_{|t| \leq 1 /\left|a_{n}\right|} t^{2} d F(t)$.

It suffices to consider the series (where we may take $a_{n} \neq 0$ )

$$
\begin{aligned}
\sum_{n} a_{n}^{2} \int_{0}^{1 /\left|a_{n}\right|} t^{2} d F(t) \leqq \sum_{n} a_{n}^{2}\left(a_{n}^{-2}-2 \int_{0}^{1 /\left|a_{n}\right|} t F(t) d t\right) \\
\quad=2 \sum a_{n}^{2} \int_{0}^{1 /\left|a_{n}\right|} t(1-F(t)) d t \leqq 2 \sum a_{n}^{2}\left(1+M \int_{1}^{1 /\left|a_{n}\right|} t^{1-p} d t\right) \\
\quad \leqq 2 \sum\left((3-p) a_{n}^{2}+N\left|a_{n}\right|^{p}\right) /(2-p)<\infty, \text { for some } N>0 .
\end{aligned}
$$

Thus (IV) $\Rightarrow$ (I).

Let $p=1$ and assume (V). Convergence of Series $\mathrm{A}$ and $\mathrm{C}$ is proved just as for $1<p<2$ above. Convergence of Series B follows directly from the latter clause of $(\mathrm{V})$.

Conversely, assume (I). Lemma 7.3(a) and (b) for $p=1$ then imply (V), and the proof of the theorem is complete.

One might ask whether the precise results in 7.2 generalize to other kinds of sequences of random variables such as orthogonal or strictly stationary ones. (III) $\Rightarrow$ (I) fails for $X_{n}$ identical and hence stationary. We shall also see that implications like (I) $\Rightarrow$ (III) fail in these cases.

If the $Y_{n}$ are random variables with $Y_{m} Y_{n}=0$ for $m \neq n$, then $\sum a_{n} Y_{n}$ converges almost surely for every sequence $\left\{a_{n}\right\}$.

Let $X_{n}$ be independent and equidistributed, $E X_{1}=0$, and $E X_{1}^{2}<\infty$. Let $Y$ be independent of the $X_{n}$ and $Z_{n}=Y X_{n}$. Then $\left\{Z_{n}\right\}$ is a strictly stationary sequence such that $\sum a_{n} Z_{n}$ converges with probability 1 for all $\left\{a_{n}\right\} \in l_{2}$, but we may have $E\left|Z_{1}\right|^{\alpha}=\infty$ for all $\alpha>0$.

8. Failure of extensions of Sazonov's theorem. If $S$ is a Hilbert space, $L$ is a continuous r.l.f. over $S$ and $A$ is Hilbert-Schmidt then $L \circ A$ is decomposable. 
Although one can define Hilbert-Schmidt operators on Banach spaces as a class including the nuclear operators, several workers had found that the statement above does not carry over to general Banach spaces. Here, answering a question of L. Schwartz, we show that it is not enough even for $A$ to be nuclear. He and A. Badrikian have recently shown that it suffices for $A$ to be only a little better than nuclear.

(8.1) Proposition. There exist a separable Banach space $X$, a nuclear operator $A$ from $X$ into itself, and a continuous linear map $L$ from $X$ into an $\mathscr{F}(P)$ such that $L \circ A$ is not decomposable.

Proof. We form the direct sum $X=c_{0} \oplus l_{1}$ with norm

$$
\left\|\left\{u_{n}\right\},\left\{v_{n}\right\}\right\|=\left\|\left\{u_{n}\right\}\right\|_{\infty}+\left\|\left\{v_{n}\right\}\right\|_{1} .
$$

Let $A$ map $c_{0}$ into $l_{1}$ by

$$
A\left(\left\{u_{n}\right\}\right)=\left\{u_{n} / n(\log n)^{2}\right\} .
$$

Letting $A=0$ on $l_{1}$ we obtain an operator from $X$ into $X$ which is clearly nuclear.

Let $Y_{n}$ be independent random variables over a probability space $(\Omega, \mathscr{B}, P)$ with common probability distribution $P$ the Cauchy density $d P=d x / \pi\left(1+x^{2}\right)$.

We define $L: l_{1} \rightarrow \mathscr{F}(P)$ by $L\left(\left\{v_{n}\right\}\right)=\sum v_{n} Y_{n}$. We extend $L$ to $X$, letting $L=0$ on $c_{0}$.

Theorem 7.2 implies that the series defining $L$ converges in probability, since the Cauchy distribution is weakly 1 -finite and symmetric.

Now $L$ is continuous from $l_{1}$ into $(\mathscr{F}(P), \mathscr{T}(P))$ by 7.1. If $L \circ A$ were decomposable, then since the dual space of $c_{0}$ is $l_{1}$, the series $\sum\left|Y_{n}\right| / n(\log n)^{2}$ would converge with probability 1 . This is false by the three-series theorem, Series B:

$$
\begin{aligned}
\sum_{n} E\left(\left(\left|Y_{n}\right| / n(\log n)^{2}\right)^{1}\right) & \geqq \sum_{n} \int_{0}^{n(\log n)^{2}} x d x / 3\left(1+x^{2}\right) n(\log n)^{2} \\
& \geqq \sum_{n} \log \left(1+n^{2}(\log n)^{4}\right) / 6 n(\log n)^{2} \geqq \sum_{n} 1 / 6 n(\log n)=+\infty .
\end{aligned}
$$

The proof is complete.

If $S, M$ and $N$ are Banach spaces, $A$ is nuclear from $S$ to $M$, and $B$ is bounded from $M$ to $N$ then clearly $B A$ is nuclear from $S$ to $N$. The following is known and easy to prove.

(8.2) Proposition. Suppose A is a nuclear operator from a Banach space $S$ into a space $L^{1}(\Omega, Q)$ of integrable functions over measure space $(\Omega, Q)$. Then $A$ is decomposable.

We call two measures $P$ and $Q$ equivalent, $P \approx Q$, if each is absolutely continuous with respect to the other. If $P \approx Q$ then the spaces $\mathscr{F}(P)$ and $\mathscr{F}(Q)$ coincide. If $(\Omega, P)$ is the unit interval with Lebesgue measure, $0<p<1$, and

$$
F=\left\{f \in \mathscr{F}(P): \int|f|^{p} \leqq 1\right\},
$$


then the closed convex hull of $F$ in $\mathscr{F}(P)$ is all of $\mathscr{F}(P)$ and is hence unbounded. Thus one might conjecture that any bounded closed convex set in $\mathscr{F}(P)$ is included in the unit ball of $L^{1}(\Omega, Q)$ for some $Q \approx P$. But this is not true:

(8.3) Proposition. Let $(\Omega, P)$ be the unit interval with Lebesgue measure (or any isomorphic measure space). Then there exists a closed, convex, bounded set $B \subset \mathscr{F}(P)$ which is not included in the unit ball of $L^{1}(\Omega, Q)$ for any $Q \approx P$.

Proof. Since the conclusion is unaffected by measure isomorphisms we take $(\Omega, P)$ as constructed in the proof of 8.1 . For the isomorphism of this with the unit interval see Halmos [16, $\S 41$, Theorem $C$, p. 173]. Let $C$ be the image of the unit ball of $c_{0}$ under the map $L: c_{0} \rightarrow \mathscr{F}(P)$ of 8.1 . Then $C$ is convex, symmetric and bounded. Let $B$ be the closure of $C$. $B$ is closed, convex, symmetric and bounded.

Suppose $Q \approx P$ and $\int|f| d Q \leqq 1$ for all $f \in C$. Then $L \circ A$ is a nuclear map of $l_{1}$ into $L^{1}(\Omega, Q)$, so by 8.2 it is decomposable, contradicting 8.1 . The proof is complete.

A closed convex symmetric bounded set in $\mathscr{F}(P)$ is the unit ball of a Banach space $T$ with a continuous injection $i$ of $T$ into $\mathscr{F}(P)$. Thus we can reformulate 8.3 as follows:

(8.4) There is a Banach space $T$ with a continuous injection $i$ into an $\mathscr{F}(P)$ such that $i(T)$ is not included in $L^{1}(\Omega, Q)$ for any $Q \approx P$.

Proof. Under the given conditions $i(T) \subset L^{1}(\Omega, Q)$ implies that $i$ is bounded from $M$ into $L^{1}(\Omega, Q)$, which need not be true according to 8.3. Q.E.D.

(8.5) Proposition. Let $A$ be a nuclear operator from a Banach space $S$ into a Hilbert space $H$ and let $L$ be continuous and linear from $H$ into an $\mathscr{F}(P)$. Then $L \circ A$ is decomposable on $S$.

Proof. Let $A(x)=\sum a_{n} b_{n}(x) f_{n}, \quad b_{n} \in S^{\prime}, \quad\left\|b_{n}\right\|^{\prime} \leqq 1, \quad a_{n}>0, \quad \sum a_{n}<\infty, f_{n} \in H$, $\left\|f_{n}\right\| \leqq 1$. The map

$$
A_{1}: x \rightarrow\left\{a_{n}^{1 / 2} b_{n}(x)\right\}
$$

is bounded from $S$ into the Hilbert space $l_{2}$, and

$$
A_{2}:\left\{y_{n}\right\} \rightarrow \sum a_{n}^{1 / 2} y_{n} f_{n}
$$

is a Hilbert-Schmidt operator from $l_{2}$ into $H$. Thus $L \circ A_{2}$ is decomposable on $l_{2}$ by 5.2. Hence $L \circ A=L \circ A_{2} \circ A_{1}$ is decomposable on $S$. Q.E.D.

The following answers a question of $\mathrm{L}$. Le Cam.

(8.6) Proposition. There exists a Banach space $X$, a decomposable map $A$ from $X$ into a space $H=L^{2}(S, v)$, and an isometry (unitary) $U$ from $H$ into a space $L^{2}(\Omega, P)$, such that $U \circ A$ is not decomposable. Specifically we can take $X=l_{1}, S=c_{0}, \nu$ to be concentrated in a compact subset of $S$, and $A$ the natural identification of $l_{1}$ with linear functionals on $c_{0}$. 
Proof. Let $y_{n}$ be independent random variables where $y_{n}$ is uniformly distributed over the interval

$$
\left\{y:|y| \leqq 3^{1 / 2} n^{-1 / 4}\right\} .
$$

Then $E y_{n}=0, E y_{n}^{2}=n^{-1 / 2}$. The distribution of the sequence $\left\{y_{n}\right\}$ is a Borel measure $\nu$ concentrated in a compact subset $K$ of $c_{0}$.

The natural map $A$ is clearly decomposable.

Let $Z_{n}$ be independent random variables over some probability space $(\Omega, P)$ with

$$
P\left(Z_{n} \neq 0\right) / 2=P\left(Z_{n}=n^{1 / 4}\right)=P\left(Z_{n}=-n^{1 / 4}\right)=1 / 2 n .
$$

Then $E Z_{n}=0, E Z_{n}^{2}=n^{-1 / 2}$, and $P\left(\sup Z_{n}=\infty\right)=1$.

Let $U\left(y_{n}\right)=Z_{n}$. Then $U$ extends to a linear isometry of $L^{2}(S, \nu)$ into $L^{2}(\Omega, P)$, but clearly $U \circ A$ is not decomposable. Q.E.D.

Alternately we could let $y_{n}$ be independent Gaussian random variables with mean 0 and variances $n^{-1 / 2}$ and obtain the same results except that $\nu$ would not be concentrated in a compact set.

Examples like those in 8.6 have been known for some time to V. N. Sudakov and A. M. Veršik but apparently not published in detail (see the sentence after the statement of Theorem 5 of [24]). They pose the following question: given a topological linear space $(S, \mathscr{T})$ is there a topology $\mathscr{U}$ on $S$ such that an r.l.f. on $S$ is canonical for $\mathscr{T}$ if and only if it is continuous for $\mathscr{U}$ ? If $(S, \mathscr{T})$ is a Hilbert (or countably-Hilbert) space, the answer is "yes," and $\mathscr{U}$ may be taken as the weakest topology for which all Hilbert-Schmidt operators are continuous from $(S, \mathscr{U})$ to $(S, \mathscr{T})([14],[22])$. L. Gross points out that $\mathscr{U}$ may also be taken to be the stronger topology on $S$ defined by all pseudo-norms which are "measurable" in his sense ([14, p. 28], [8, Theorem 4.6, Proposition 6.7, and remarks after 6.9]).

Sudakov and Veršik observe that if $(S, \mathscr{T})$ is a Banach space, the topology $\mathscr{U}$ need not exist. For any r.l.f. with a version $L$ there is a weakest topology $\mathscr{T}_{L}$ for which it is continuous. Then $\left(S, \mathscr{T}_{L}\right)$ is a topological linear space (not necessarily locally convex). If $\mathscr{U}$ exists and $L$ is decomposable, then $\mathscr{T}_{L} \subset \mathscr{U}$. Thus if $M$ is continuous for $\mathscr{T}_{L}$ it would be canonical. If $L$ is Gaussian, then $\mathscr{T}_{L}$ is the inner product topology defined by the covariance of $L$. The proof of 8.6 above and the remark immediately following it with $(S, \mathscr{T})=l_{1}$ show that $\mathscr{U}$ does not exist on $l_{1}$, where $M=U \circ A$.

9. $L^{p}$ maps of Banach spaces. I learned the material presented through 9.4 below from lectures by A. Pietsch and L. Schwartz; its virtues may be assumed to be theirs and its faults, mine.

Definition. Let $S$ and $T$ be Banach spaces and let $A$ be a linear map from $S$ into $T$. Let $1 \leqq p<\infty$ and let $q$ be the conjugate index $p /(p-1)$, or $\infty$ if $p=1$. We say $A \in L^{p}=L^{p}(S, T)$ if for all $s$ in $S$

$$
A(s)=\sum_{n} b_{n} g_{n}(s) t_{n}
$$


where $t_{n} \in T,\left\|t_{n}\right\| \leqq 1, g_{n} \in S^{\prime}, b_{n} \geqq 0, \sum b_{n}^{p}<\infty$, and $s \rightarrow\left\{g_{n}(s)\right\}$ is bounded from $S$ into $l_{q}$.

By Hölder's inequality, the series (9.1) converges absolutely in the Banach space $T$, uniformly for $s$ in bounded subsets of $S$. Clearly the $L^{1}$ operators are exactly the nuclear operators.

(9.2) Proposition. If $1 \leqq p<r<\infty$, then $L^{p}(S, T) \subset L^{r}(S, T)$.

Proof. Let $A \in L^{p}(S, T)$ and let (9.1) and the other conditions in the definition hold. Let $c_{n}=b_{n}^{p / r}, h_{n}=b_{n}^{(r-p) / r} g_{n}$. Then $\left\{c_{n}\right\} \in l_{r}$ and

$$
\left\{b_{n}^{(r-p) / r}\right\} \in l_{p r /(r-p)}
$$

Let $t=r /(r-1)$. Then the map $s \rightarrow\left\{h_{n}(s)\right\}$ is bounded by Hölder's inequality from $S$ into $l_{t}$. Q.E.D.

(9.3) Proposition. If $(\Omega, P)$ is a measure space and $L \in L^{p}\left(S, L^{p}(\Omega, P)\right)$ then $L$ is decomposable.

Proof. By (9.1) and the rest of the definition with $L=A$ we have

$$
E \sum_{n} b_{n}^{p}\left|t_{n}(\omega)\right|^{p}<\infty
$$

so $\left\{b_{n} t_{n}(\omega)\right\} \in l_{p}$ for almost all $\omega$ and then $s \rightarrow L(s)(\omega)$ belongs to $S^{\prime}$ by Hölder's inequality. Q.E.D.

(9.4) Proposition. An operator A from one Hilbert space $H$ to another $J$ is Hilbert-Schmidt if and only if $A \in L^{2}(H, J)$.

Proof. We can assume $H$ and $J$ are infinite-dimensional. Let $A$ be HilbertSchmidt. We choose an orthonormal set $\left\{f_{n}\right\}$ such that $A g=0$ if $g \perp f_{n}$ for all $n$. Then $\sum\left\|A f_{n}\right\|^{2}<\infty$. For any $g$ in $H$ we have

$$
A g=\sum\left(g, f_{n}\right) A f_{n}=\sum\left(g, f_{n}\right) a_{n} h_{n}
$$

where $\left\|h_{n}\right\| \leqq 1$ and $\sum\left|a_{n}\right|^{2}<\infty$, so indeed $A \in L^{2}(H, J)$.

Conversely let $A \in L^{2}(H, J)$, with (9.1) etc. holding, $g_{n}(s)=\left(s, g_{n}\right), g_{n} \in H$. Let $\left\{f_{n}\right\}$ be an orthonormal set in $H$. Then the operator $B$ from $H$ into itself defined by $B f \equiv \sum\left(f, g_{n}\right) f_{n}$ is bounded. The operator $C$ from $H$ to $J$ such that $C g \equiv \sum a_{n}\left(g, f_{n}\right) t_{n}$ is clearly Hilbert-Schmidt, and $A=C B$. Then $A^{*}=B^{*} C^{*}$ is Hilbert-Schmidt and hence so is $A$. Q.E.D.

Next we show that in one sense, 9.3 is best possible.

(9.5) Proposition. Let $(\Omega, P)$ be the unit interval with Lebesgue measure and $\mathrm{Ha}$ separable infinite-dimensional Hilbert space. Then there is a map $A$ belonging to $L^{p}\left(H, L^{1}(\Omega, P)\right)$ for all $p>1$ but which is not decomposable.

Proof. For each positive integer $r$ and $j=0,1, \ldots, 2^{r}-1$, let $F_{j r}(x)=1, j / 2^{r} \leqq x$ $<(j+1) / 2^{r}$, and $F_{j r}(x)=0$ otherwise. Let $\left\{f_{n}\right\}$ be an enumeration without repetitions 
of all the $F_{j r}$. Let $\left\{\varphi_{n}\right\}$ be an orthonormal basis of $H$ and let $A\left(\varphi_{n}\right)=f_{n}$ for all $n$. Then for $p>1$

$$
\sum_{n}\left(\int_{0}^{1}\left|f_{n}\right| d P\right)^{p}=\sum_{r=1}^{\infty} 2^{r} / 2^{r p}<\infty
$$

so $A \in L^{p}\left(H, L^{1}(\Omega, P)\right)$. However, $A$ is not decomposable because if $g_{n}=f_{n} P$-almost surely, and $B: H \rightarrow L^{1}$ is such that for each $\varphi \in H, A(\varphi)=B(\varphi) P$-almost surely, then for almost all $x$ in $\Omega$,

$$
\sup \{|B(\varphi)(x)|:\|\varphi\| \leqq 1\}=+\infty .
$$

10. Means and translates. Let $S$ be a topological linear space and let $\left(S^{a}, P\right)$ be an r.l.f. over $S$. We shall see that even if $P$ is not canonical, certain elements of $S^{a}$ naturally associated with $P$ often are continuous, at least sequentially, on $S$.

Given $x \in S^{a}$ we define the translate of $P$ by $x$ : let $P^{x}(A)=P(A-x), A \in \mathscr{B}\left(S^{a}, S\right)$. (Note that $\mathscr{B}\left(S^{a}, S\right)$ is translation-invariant.)

(10.1) Proposition. If the r.l.f. $\left(S^{a}, P\right)$ over $S$ is sequentially continuous and $P^{x}$ is not singular with respect to $P$ then $x$ is sequentially continuous on $S$.

Proof. Suppose $s_{n} \rightarrow 0$ in $S$. Let $S_{n}(y)=y\left(s_{n}\right), y \in S^{a}$. Then $S_{n} \rightarrow 0$ in $P$-probability. Hence $S_{n} \rightarrow 0$ in $P^{x}$-probability on a set with positive $P^{x}$-probability, i.e. $S_{n}(x)+S_{n} \rightarrow 0$ in $P$-probability on a set with positive $P$-probability, and so do $S_{n}(x)$, i.e. the constants $x\left(s_{n}\right) \rightarrow 0$. Q.E.D.

The above proposition is closely related to [7, Theorem 1]. Sequential continuity implies continuity, i.e. $x \in S^{\prime}$, if $S$ is metrizable or bornologic (see e.g. [5, Theorems 6.1 and 6.3]).

We shall call the r.l.f. $\left(S^{a}, P\right)$ integrable if $\int|x(s)| d P(x)<\infty$ for all $s \in S$. Then $M(s)=\int x(s) d P(x)$ is a well-defined linear functional on $S$, called the mean of $P$. The following implies some results of Ahmad [1, Proposition 7] who makes the stronger assumptions that $P$ is canonical and $S$ a metrizable space or inductive limit thereof.

(10.2) Proposition. If $S$ is barreled and $\left(S^{a}, P\right)$ is a continuous, integrable r.l.f. over $S$, then the natural map of $S$ into $L^{1}\left(S^{a}, P\right)$ is continuous so the mean $M$ is continuous.

Proof. Let $B=\left\{s: \int|x(s)| d P(x) \leqq 1\right\}$. Then $B$ is convex, symmetric, and absorbing. Suppose $s_{\alpha} \in B$ and $s_{\alpha} \rightarrow s$. Then $x\left(s_{\alpha}\right) \rightarrow x(s)$ in $P$-probability so there exist sets $A$ with $P(A)$ arbitrarily close to 1 and $\int_{A}|x(s)| d P(x) \leqq 1$. This implies $s \in B$, i.e. $B$ is closed, hence a barrel and a neighborhood of 0 . Q.E.D.

We call an r.l.f. $\left(S^{a}, P\right)$ Gaussian if its ch.f. is of the form $\chi(x)=e^{i y(x)-Q(x)}$ where $y \in S^{a}$ and $Q$ is a nonnegative definite quadratic form on $S$. Then $P$ has mean $y$ and $P=P_{1}^{v}$ where $P_{1}$ has ch.f. $e^{-Q}$. Now if $\left(S^{a}, P\right)$ is Gaussian and continuous (resp. sequentially continuous) then its mean $y$ is likewise continuous (resp. sequentially continuous) with no special assumptions about $S$. 
We mentioned above some cases in which sequential continuity implies continuity for linear functionals on $S$. Here is another one. Suppose $X$ is a complete locally convex topological linear space with a countable dense subset. Let $S=X^{\prime}$ with the weak-star topology $w(S, X)$. Then each sequentially continuous linear functional on $S$ is continuous, i.e. defined by an element of $X$ (see H. Schaefer, Topological vector spaces, Macmillan, New York, 1966, p. 150, Corollary 3). This need not be true if $X$ has no countable dense set, even if it is a Banach space; see B. Kripke, One more reason why sequences are not enough, Amer. Math. Monthly 74 (1967), 563-565.

11. Stochastic processes and random distributions. Let $R$ be the real line and $(\Omega, P)$ a probability space. A function $x(\cdot, \cdot)$ from $T \times \Omega$ to $R$, where $T \subset R$, is called a stochastic process if $x(t, \cdot)$ is measurable on $\Omega$ for each fixed $t \in T . x(\cdot, \cdot)$ is called measurable if it is measurable from $T \times \Omega$ into $R$ for the usual Borel $\sigma$-algebras in $R$ and $T$.

A stochastic process $x(\cdot, \cdot)$ is equivalent to a version $L$ of an r.l.f. over the linear space $F(T, R)$ of functions from $T$ to $R$ with finite support, by

$$
L(f)(\omega)=\sum_{t} f(t) x(t, \omega) .
$$

Note that $F(T, R)^{a}$ identifies naturally with the space $R^{T}$ of all real-valued functions on $T$. This relationship holds for an arbitrary abstract set $T$. For $T=R$ we can also seek a version $L_{x}$ of an r.l.f. with

$$
L_{x}(f)(\omega)=\int_{-\infty}^{\infty} f(t) x(t, \omega) d t
$$

Suppose that $f \in S$ where $S$ is a suitable space of "test functions," e.g. $C^{\infty}$ functions with compact support. Then (11.2) will make sense if $x(\cdot, \cdot)$ has locally integrable sample functions, i.e.

$$
\int_{a}^{b}|x(t, \omega)| d t<\infty
$$

for almost all $\omega$, and any finite $a<b$. However, this does not assure that $L_{x}(f)(\cdot)$ is measurable, and in fact the locally integrable functions have outer measure 1 for every probability measure $P$ on $\mathscr{B}(F(R, R))$ in $F(R, R)^{a}$. (If $A \in \mathscr{B}(F(R, R)$ ), then whether $f \in A$ or not depends only on the values of $f$ at countably many points. Hence $A$ cannot be disjoint from the locally integrable functions unless it is empty. For the classes of continuous functions, or functions with only jump discontinuities, the situation is different.)

We show that measurability of $x(\cdot, \cdot)$ is a sufficient additional assumption, using

(11.3) Proposition. Suppose $(S, \mathscr{B}, \mu)$ is a finite measure space and $\mathscr{V}$ is a $\sigma$-algebra of subsets of a set $T$. Let $x(\cdot, \cdot)$ be a product measurable function on $S \times T$. Let $g(t)=\int x(s, t) d \mu(s)$. Then the domain of $g$ belongs to $\mathscr{V}$ and $g$ is $\mathscr{V}$-measurable on its domain. 
Proof. If $x \geqq 0$ on $S \times T$ the result follows by taking a sequence of simple functions increasing up to $x$ (cf. Halmos [16, Theorem A, p. 143, Theorem B, p. 147], putting the zero measure on $T)$. In general we can let $y=\max (x, 0), z=-\min (x, 0)$, $h=\int y d \mu, j=\int z d \mu$, so $g=h-j$, being defined and finite if and only if both $h$ and $j$ are, so the result for $y$ and $z$ implies it for $x$. Q.E.D.

We infer that a measurable stochastic process on $R$ with locally integrable sample functions defines by (11.2) an r.l.f. on the space of bounded measurable functions on $R$ with compact support, or any smaller function space.

\section{REFERENCES}

1. Salah Ahmad, Eléments aléatoires dans les espaces vectoriels topologiques, Ann. Inst. $\mathrm{H}$. Poincaré Sect. B 2 (1965/66), 95-135.

2. S. Banach, Théorie des opérations linéaires, PWN, Warsaw, 1932; reprint, Chelsea, New York, 1955.

3. N. Bourbaki, Espaces vectoriels topologiques, Hermann, Paris, 1955, Chapters III-V.

4. Pierre Cartier, Processus aléatoires généralisés, Séminaire Bourbaki, 16e année: 1963/64, Fasc. 3, Exposé 272, Secretariat mathématique, Paris, 1964.

5. R. M. Dudley, On sequential convergence, Trans. Amer. Math. Soc. 112 (1964), 483-507.

6. - Singular translates of measures on linear spaces, $\mathrm{Z}$. Wahrscheinlichkeitstheorie und Verw. Gebiete 3 (1964), 128-137.

7. - Singularity of measures on linear spaces, Z. Wahrscheinlichkeitstheorie und Verw. Gebiete 6 (1966), 129-132.

8. - The sizes of compact subsets of Hilbert space and continuity of Gaussian process, J. Functional Anal. 1 (1967), 290-330.

9. James Dugundji, Topology, Allyn and Bacon, Boston, Mass., 1966.

10. N. Dunford and J. Schwartz, Linear operators, Part I, Interscience, New York, 1958.

11. J. Feldman, On extending complex quasimeasures, Theor. Probability Appl. 10 (1965), 345-348.

12. I. M. Gelfand and N. Ya. Vilenkin, Generalized functions, Vol. 4: Some applications of harmonic analysis, Fizmatgiz, Moscow, 1961; English transl., Academic Press, New York, 1964.

13. R. K. Getoor, On characteristic functions of Banach space valued random variables, Pacific J. Math. 7 (1957), 885-896.

14. Leonard Gross, Harmonic analysis on Hilbert space, Mem. Amer. Math. Soc. No. 46 (1963).

15. Alexandre Grothendieck, Produits tensoriels topologiques et espaces nucléaires, Mem. Amer. Math. Soc. No. 16 (1955). (Erratum: Ann. Inst. Fourier (Grenoble) 6 (1955/56), 117120.)

16. P. R. Halmos, Measure theory, Van Nostrand, Princeton, N. J., 1950.

17. E. Hewitt and K. A. Ross, Abstract harmonic analysis, Vol. I: Structure of topological groups. Integration theory, group representations, Academic Press, New York, 1963.

18. A. N. Kolmogorov, A note to the papers of R. A. Minlos and V. Sazonov, Teor. Verojatnost. i Primenen. 4 (1959), 237-239= Theor. Probability Appl. 4 (1959), 221-223.

19. M. Loève, Probability theory, 2nd ed., Van Nostrand, Princeton, N. J., 1960.

20. R. A. Minlos, Generalized random processes and their extension to measures, Trudy Moskov. Mat. Obšč. 8 (1959), 497-518; Selected Transl. Math. Stat. and Prob., vol. 3, Amer. Math. Soc., Providence, R. I., 1963, pp. 291-314. 
21. F. Riesz and B. Sz.-Nagy, Functional analysis, Ungar, New York, 1955.

22. V. Sazonov, On characteristic functionals, Teor. Verojatnost. i Primenen. 3 (1958), 201205= Theor. Probability Appl. 3 (1958), 188-192.

23. I. E. Segal, Abstract probability spaces and a theorem of Kolmogoroff, Amer. J. Math. 76 (1954), 721-732.

24. V. N. Sudakov and A. M. Veršik, Topological questions in the theory of measures in linear spaces, Uspehi Mat. Nauk 17 (1962), no. 4 (106), 217-219. (Russian)

25. V. S. Varadarajan, Measures on topological spaces, Mat. Sb. 55 (97) (1961), 35-100= Amer. Math. Soc. Transl. (2) 48 (1965), 161-228.

26. S. Bochner, Harmonic analysis and the theory of probability, Univ. of California Press, Berkeley, Calif., 1955.

Massachusetts Institute of Technology,

CAMBridge, MassachusetTS 